\title{
Donor characteristics and intra-operative total nucleated cell count influence hematopoietic progenitor yield of healthy donor bone marrow grafts
}

\author{
Jacob Kalin $^{1}$, Anh Thy Nguyen ${ }^{1}$, and Benjamin Oshrine ${ }^{1}$ \\ ${ }^{1}$ Johns Hopkins All Children's Hospital
}

October 22, 2020

\begin{abstract}
Background: Bone marrow graft cell content impacts engraftment potential after allogeneic hematopoietic cell transplantation (alloHCT). Surrogates such as intra-operative total nucleated cell count (ioTNC), are of unclear utility in predicting final graft characteristics. In addition, demographic and clinical factors may influence graft cellular profile and recipient engraftment. Procedure: We retrospectively reviewed marrow harvests at our institution performed between 2009 and 2019. During this time, an ioTNC was measured after $50 \%$ of the projected final graft volume was collected. Regression models were used to assess associations between ioTNC (cells/ $/ \mathrm{L})$ and final graft CD34+ cells $/ \mathrm{mL}$, and between graft and donor characteristics and final graft CD34+ cells/mL. Results: Fifty-three marrow harvests and donor-recipient pairs were analyzed. Median (range) donor and recipient ages were 13 (0.7-28) years and 9 (0.2-21) years, respectively. The median ratio of donor/recipient weight was 1.225 (range 0.31-7.13). Median total volume of harvested marrow was $15.3 \mathrm{ml} / \mathrm{kg}$ (range $4.3-20.4 \mathrm{ml} / \mathrm{kg}$ ) of donor weight and $19.4 \mathrm{ml} / \mathrm{kg}$ (range $4.7-87.4 \mathrm{ml} / \mathrm{kg}$ ) of recipient weight. Median ioTNC was $20930 / \mu \mathrm{L}$ (range $6600-44310 / \mu \mathrm{L}$ ) or $2.1 \times 109 / \mathrm{mL}$, corresponding to median predicted final graft TNC of $3.59 \mathrm{x} 108 / \mathrm{kg}$ recipient weight (range 1.28-19.42x108). Simple linear regression between ioTNC and CD34+ cells/mL resulted in an $\mathrm{R} 2$ of 0.42 . LASSO regression produced a moderately predictive model consisting of ioTNC, donor age, and donor weight (adjusted R2=0.7) of final graft CD34+ cells $/ \mathrm{mL}$. Conclusions: ioTNC and certain donor characteristic correlate moderately well with marrow product CD34+ cells/m, potentially informing donor selection and marrow procurement strategies.
\end{abstract}

\section{INTRODUCTION}

Bone marrow remains the most commonly used stem source for allogeneic hematopoietic cell transplantation (alloHCT) in pediatric recipients (1). The cellular composition of allogeneic bone marrow grafts is known to have a direct impact on outcomes after alloHCT, including the likelihood and timing of engraftment, kinetics of immune reconstitution, incidence of graft-versus-host disease (GVHD), and other transplant outcomes (24). Therefore, optimizing the bone marrow graft harvest process is critical to the success of alloHCT. Typically, the total nucleated cell (TNC) and/or CD34+ cell quantities (in terms of recipient weight) are used to assess the adequacy of a graft as proxies for hematopoietic progenitor cell content, and influence graft collection strategies. However, concerns regarding optimal graft characteristics are balanced against ensuring the safety of volunteer donors, particularly when these donors are children (5).

Established standards limit the maximum amount of marrow $(20 \mathrm{ml} / \mathrm{kg}$ of donor weight $)$ that can be safely harvested from pediatric donors to minimize complications (6). Various procedural techniques can help improve collection yields $(7,8)$. When the recipient is of comparable or lesser weight than the donor, harvest targets can be well below the maximum allowable collection volume; however, when the recipient is significantly larger than the donor, achieving minimum cell dose with a bone marrow graft can be challenging. 
The TNC and CD34+ yields vary based on multiple donor factors, including age and peripheral leukocyte count among others, and can be difficult to predict (8-10). Therefore, it is common practice to assess product cell counts during the actual harvest procedure, by obtaining an intraoperative TNC (ioTNC). This has the intent of providing real-time information on cell yield to guide harvest volume targets, avoiding insufficient collections as well as minimizing harvest volumes to optimize donor safety. However, in practice, obtaining ioTNC has questionable utility, and it is uncertain whether this correlates with final product cell counts and clinical outcomes. We retrospectively reviewed bone marrow harvests performed at our institution over an 11-year period to assess the impact of donor factors and ioTNC on final graft characteristics and recipient clinical outcomes.

\section{MATERIALS AND METHODS}

The study was a retrospective review of all allogeneic bone marrow collections performed from January 2009 to December 2019. The data analysis was limited to donor-recipient pairs for whom ioTNC was available. Autologous collections ("back-up" grafts) and collections in which hematopoietic growth factor was used for donor stimulation were excluded. Information on donor and recipient was extracted manually from the electronic medical record. During the study period, the standard of care was to perform ioTNC after $50 \%$ of the projected final graft volume (based on donor/recipient weights) was collected, to guide collection volume targets. Predicted final product TNC was calculated by multiplying the ioTNC concentration by the end volume, after converting the ioTNC units.

The primary outcome variable of interest was the final harvest product CD34+ cell count. Secondary outcomes included engraftment kinetics of neutrophils and platelets and overall engraftment. The association between ioTNC and final graft CD34+ count was assessed by simple and multivariate linear regression models. CD34+ cell count and continuous independent variables were log-transformed for regression modeling to produce normally distributed residuals and facilitate interpretation. Potential predictors were screened for inclusion in multivariate models using Least Absolute Shrinkage and Selection Operator (LASSO) regression with the Corrected Akaike Information Criterion (AICc). The model produced from LASSO regression was further evaluated for multicollinearity based on variance inflation factors [?]10. All analyses were conducted using SAS version 9.4 (SAS Institute, Cary, NC).

\section{RESULTS}

\section{Donor and Recipient Characteristics}

During the study period a total of 74 bone marrow collections were performed, of which 14 were autologous and an additional 7 lacked ioTNC data, leaving 53 donor-recipient pairs for analysis. Patient, donor, and transplant characteristics are summarized in Table 1. Hematologic malignancy (40.7\%) and aplastic anemia/bone marrow failure $(20.8 \%)$ were the most common indications for transplant, and most patients received myeloablative conditioning (64.7\%). The median donor and recipient ages were 13 (range 0.7-28) years and 9 (range 0.2-21) years, respectively. The median ratio of donor/recipient weight was 1.225 (range 0.31-7.13). While 11 donors weighed less than the recipient, only 3 donors were $<75 \%$ of recipient weight.

\section{Harvest characteristics}

The median volume of marrow collected was $15.3 \mathrm{ml} / \mathrm{kg}$ of donor weight (range $4.3-20.4 \mathrm{ml} / \mathrm{kg}$ ) and $19.4 \mathrm{ml}$ per kilogram of recipient weight (krw) (range $4.7-87.4 \mathrm{ml} / \mathrm{kg}$ ). The median ioTNC was 20,930 cells $/ \mu \mathrm{L}$ (range 6600-44310 cells/ $\mu \mathrm{L}$ ), corresponding to median predicted final graft TNC of $3.59 \times 10^{8} / \mathrm{krw}$ (range 1.3-19.4 $\left.\times 10^{8}\right)$. Of the 6 grafts with projected $\mathrm{TNC}<2 \times 10^{8}$ cells $/ \mathrm{krw}$, all had final CD34+ cell count greater than $2 \times 10^{6} / \mathrm{krw}$. Donor complications were minimal; no healthy donors required blood product support, and 2 required additional care for anesthesia- or procedure-related issues (nausea/vomiting and pain).

\section{Engraftment}

Three patients $(5.7 \%)$ experienced graft failure, with predicted TNC doses of $2.4 \times 10^{8} / \mathrm{krw}, 14.5 \times 10^{8} / \mathrm{krw}$, and $11.2 \times 10^{8} / \mathrm{krw}$, respectively; as well as actual CD $34+$ cell doses of $12 \times 10^{6} / \mathrm{krw}, 15.9 \times 10^{6} / \mathrm{krw}$, and 
$29.1 \times 10^{6} / \mathrm{krw}$, respectively. Of engrafting patients, neutrophil and platelet engraftment occurred at a median of 18 (range 8-27) days and 26 (12-160) days, respectively. ioTNC did not correlate meaningfully with day of engraftment for either ANC or platelets (Figure 1).

Relationship between donor/collection factors and final graft characteristics

Simple linear regression between ioTNC and CD34+cells/mL result in an $\mathrm{R}^{2}$ of 0.42 (Figure 2). Of donor and patient variables analyzed, ioTNC, pre-collection donor total leukocyte count and absolute lymphocyte count correlated positively with final graft CD34 count, while donor weight, height, body surface area, and male gender correlated inversely (Table 2 and Figure 3). LASSO regression identified ioTNC, donor age, and donor weight as potential predictors. Donor leukocyte count was highly correlated with ioTNC (Pearson correlation coefficient $=0.94$ ) and thus was removed from the model to prevent multicollinearity. A model with ioTNC, donor age, and donor weight is moderately predictive (adjusted $\mathrm{R}^{2}=0.7$ ) of final graft CD34+ cells $/ \mathrm{mL}$ (Table 2). Since donor weight was highly correlated with donor BSA (Pearson's coefficient=0.99), a model with ioTNC, donor age, and donor BSA produced similar results (adjusted $\mathrm{R}^{2}=0.71$ ). Both models show that a $10 \%$ increase in ioTNC corresponded to approximately a $6 \%$ increase in final graft CD $34+/ \mathrm{mL}$.

\section{DISCUSSION}

Procurement of a quality bone marrow graft for alloHCT is of critical importance, both to optimize the clinical outcomes of the recipient, and ensure the safety of the healthy volunteer donor. While technical maneuvers can optimize hematopoietic progenitor cell yield, many practitioners utilize intra-operative cell counts to guide collection volume targets and to provide real-time information regarding quality of the collection. However, information on the value of this practice is lacking. We undertook a review of 11 years of related bone marrow graft procurement at our institution, during which time it was standard practice to obtain an ioTNC after half of the projected collection volume had been reached. The ioTNC correlated moderately with final graft CD34+cells/krw, an accepted measure of graft adequacy that is known to correlate with engraftment potential, suggesting that this practice has merit in guiding collection volume requirements.

In addition, our findings are consistent with prior reports that donor characteristics of age, pre-operative peripheral leukocyte count, and anthropometric factors influence hematopoietic progenitor cell yield. While Anthias et al did not find donor factors to be predictive of graft characteristics, graft adequacy was assessed based on TNC/krw (9). This incorporates recipient weight in a way that may overshadow relevant donor features. Indeed, Wang et al reported on a large sample of marrow harvests and found that donor leukocyte count strongly correlated with harvest product TNC density (8). This study also reported a strong correlation between midway marrow cell density (comparable to ioTNC in our report) and final marrow cell density - both measured by $\mathrm{TNC} / \mathrm{ml}$ - analogous to our findings. However, in their cohort, donor weight positively correlated with marrow harvest TNC cell density, contrary to our findings. In a study evaluating marrow harvest characteristics from children undergoing autologous marrow collections in preparation for gene therapy, Tucci et al reported similar findings to our cohort, with final graft CD34+ cell count correlating inversely with weight and age (11), the latter of which was also seen in a report of the effect of G-CSF priming on marrow cell counts (10).

Our study suggests that ioTNC correlates positively with final product CD34+ cell concentrations, and may provide clinical utility in estimating projected harvest volumes during the graft procurement process. However, in this cohort, inadequate hematopoietic progenitor yields resulting in impaired engraftment or slow engraftment kinetics was not observed. In addition, pre-collection donor characteristics (age, anthropometrics and peripheral leukocyte count) were useful in predicting graft progenitor cell content, and can be used in donor selection algorithms when multiple donor options are available. This study has important limitations, including its retrospective nature, relatively small sample size, and lack of information about intra-operative technique (e.g. volume per aliquot of marrow harvested), as technical factors are known to play a role in harvest yields (12).

\section{REFERENCES}


1. D'Souza, A, Fretham C, Lee SJ, et al. Current Use of and Trends in Hematopoietic Cell Transplantation in the United States. Biol Blood Marrow Transplant. 2020 May 11:S1083-8791(20)30225-1, doi: 10.1016/j.bbmt.2020.04.013, PMID 32438042.

2. Paulin, T. Importance of bone marrow cell dose in bone marrow transplantation. Clin Transplant; 6(1): 48-54.

3. Sierra J, Storer B, Hansen J, et al. Transplantation of marrow cells from unrelated donors for treatment of high-risk acute leukemia: the effect of leukemic burden, donor HLA-matching, and marrow cell dose.Blood ; 1997;89:4226-4235.

4. Rocha V, Labopin M, Gluckman E, et al. Relevance of bone marrow cell dose on allogeneic transplantation outcomes for patients with acute myeloid leukemia in first complete remission: results of a European survey. J Clin Oncol . 2012;20:4324-4330.

5. Pulsipher M, Logan B, Kiefer D, et al. Higher Risks of Toxicity and Incomplete Recovery in 13- to 17-Year-Old Females after Marrow Donation: RDSafe Peds Results. Biol Blood Marrow Transplant . 2019;25:955-964.

6. Yabe M, Morimoto T, Shimizu T, et al. Feasibility of marrow harvesting from pediatric sibling donors without hematopoietic growth factors and allotransfusions. Bone Marrow Transplant . 2014;49:921-6.

7. Bacigalupo A, Tong J, Piaggio G, et al. Bone marrow harvest for marrow transplantation: effect of multiple small $(2 \mathrm{ml})$ or large $(20 \mathrm{ml})$ aspirates. Bone Marrow Transplant . 1992;9: 467-470.

8. Wang T, Chu S, Chen S, et al. The effect of different harvest strategies on the nucleated cell yields of bone marrow collection.Biol Blood Marrow Transplant. 2011;17:351-355.

9. Anthias C, Billen A, Arkwright R, et al. Harvests from bone marrow donors who weigh less than their recipients are associated with a significantly increased probability of a suboptimal harvest yield. Transfusion. 2016;56:1052-1057.

10. Furey A, Rastogi S, Prince R, et al. Bone Marrow Harvest in Pediatric Sibling Donors: Role of Granulocyte Colony-Stimulating Factor Priming and CD34+ Cell Dose. Biol Blood Marrow Transplant. 2017;24:324-329.

11. Tucci F, Frittoli M, Barzaghi F, et al. Bone marrow harvesting from paediatric patients undergoing haematopoietic stem cell gene therapy.Bone Marrow Transplant. 2019;54:1995-2003.

12. Hequet, O. Hematopoietic stem and progenitor cell harvesting: technical advances and clinical utility. J Blood Med. 2015;6:55-67.

\section{FIGURE LEGEND}

Figure 1. Unadjusted association between intraoperative TNC with day of ANC and platelet engraftment

Figure 2. Unadjusted association between intraoperative TNC and final CD34+ cell count

Figure 3. Unadjusted associations between preoperative donor ANC, ALC, and WBC with intraoperative TNC and final CD34+ cell count

\section{Hosted file}

Table 1.pdf available at https://authorea.com/users/369391/articles/488265-donorcharacteristics-and-intra-operative-total-nucleated-cell-count-influence-hematopoieticprogenitor-yield-of-healthy-donor-bone-marrow-grafts

\section{Hosted file}

Table 2.pdf available at https://authorea.com/users/369391/articles/488265-donorcharacteristics-and-intra-operative-total-nucleated-cell-count-influence-hematopoieticprogenitor-yield-of-healthy-donor-bone-marrow-grafts

\section{Hosted file}

Table 3.pdf available at https://authorea.com/users/369391/articles/488265-donorcharacteristics-and-intra-operative-total-nucleated-cell-count-influence-hematopoietic- 
progenitor-yield-of-healthy-donor-bone-marrow-grafts
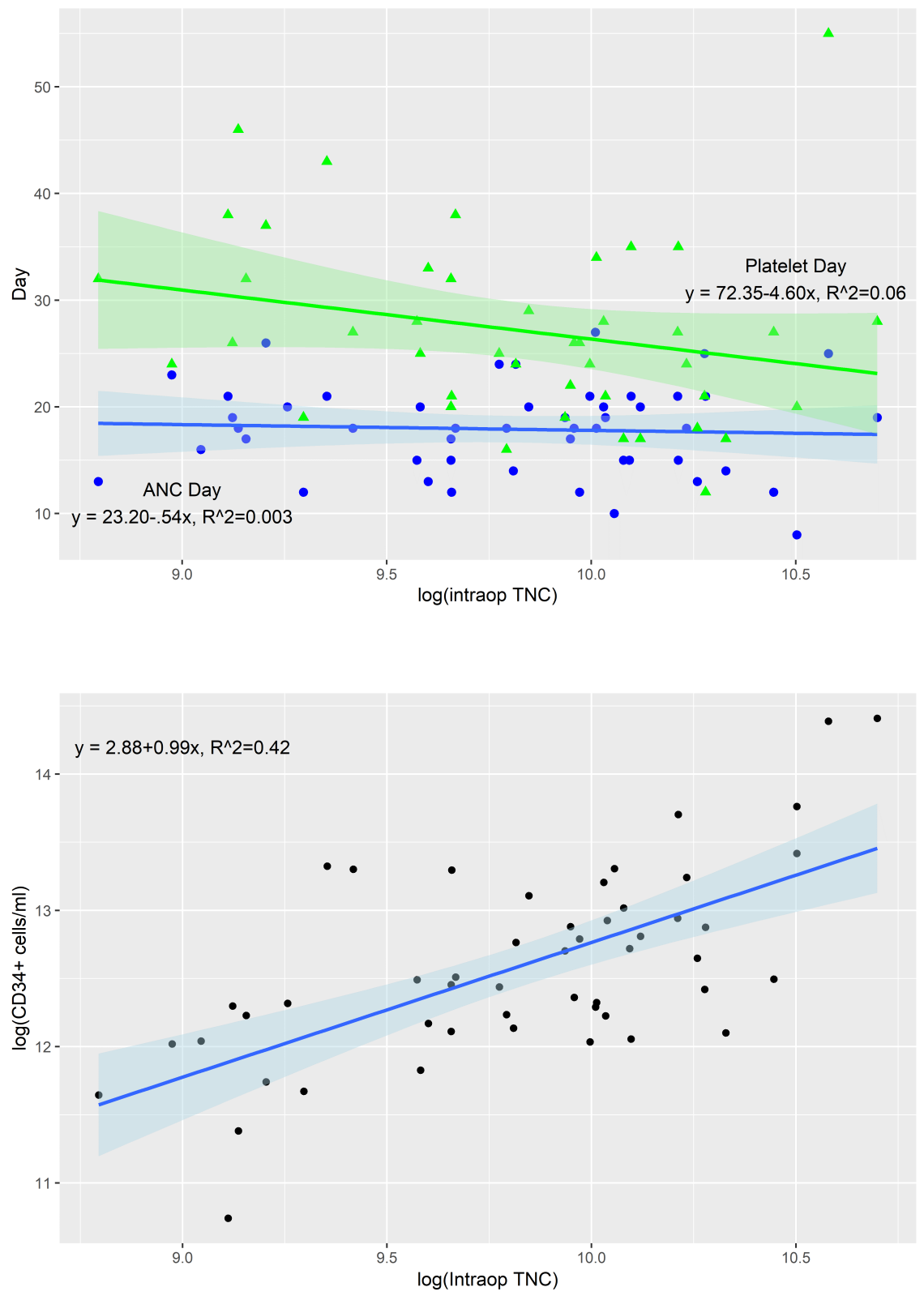

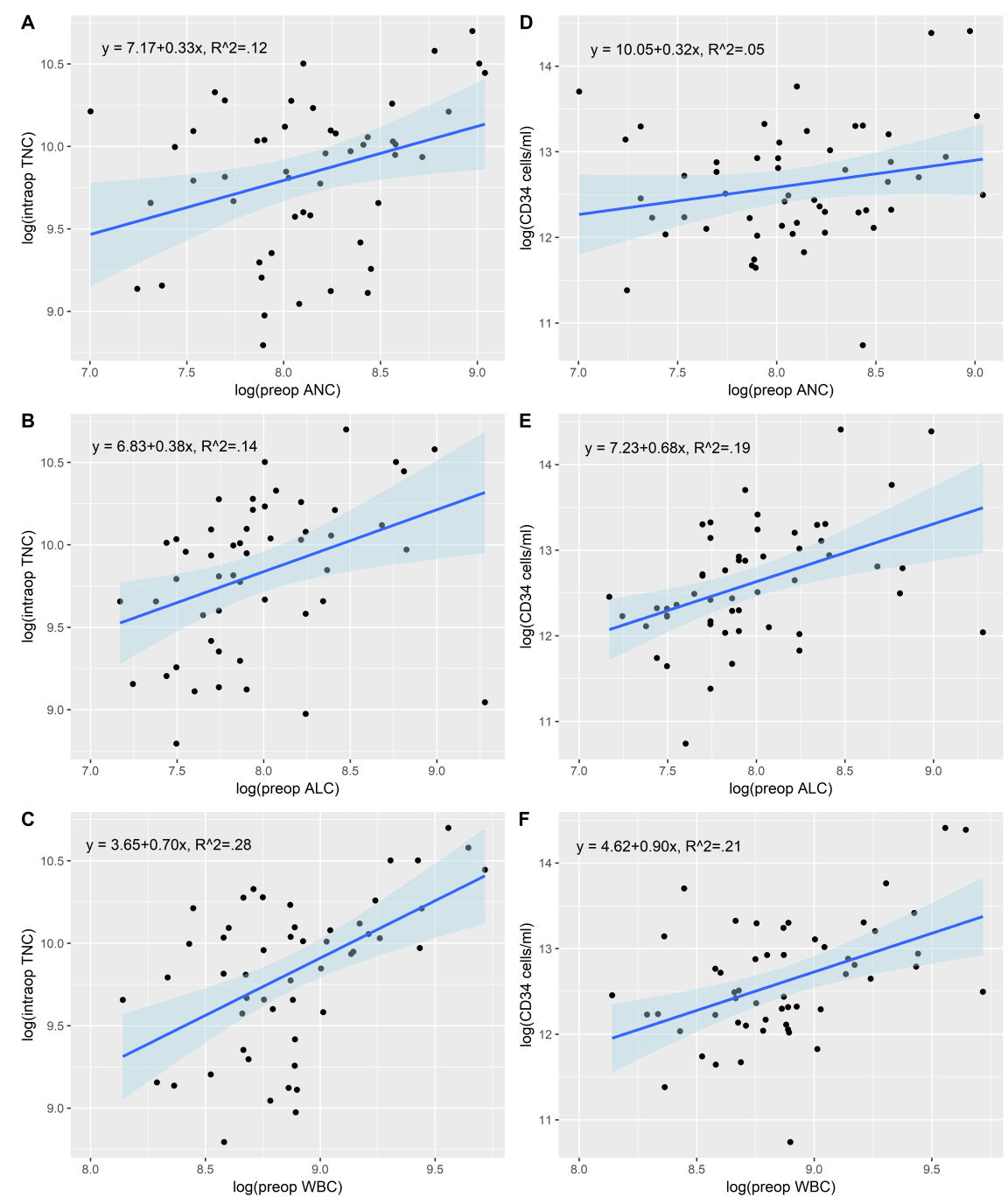